\title{
Human Error and the International Space Station: Challenges and Triumphs in Science Operations
}

\author{
Samantha S. Harris* and Beau C. Simpson ${ }^{\dagger}$ \\ NASA Marshall Space Flight Center, Huntsville, AL, 35812
}

\begin{abstract}
Any system with a human component is inherently risky. Studies in human factors and psychology have repeatedly shown that human operators will inevitably make errors, regardless of how well they are trained. Onboard the International Space Station (ISS) where crew time is arguably the most valuable resource, errors by the crew or ground operators can be costly to critical science objectives. Operations experts at the ISS Payload Operations Integration Center (POIC), located at NASA's Marshall Space Flight Center in Huntsville, Alabama, have learned that from payload concept development through execution, there are countless opportunities to introduce errors that can potentially result in costly losses of crew time and science. To effectively address this challenge, we must approach the design, testing, and operation processes with two specific goals in mind. First, a systematic approach to error and human centered design methodology shoul d be implemented to minimize opportunities for user error. Second, we must assume that human errors will be made and enable rapid identification and recoverability when they occur. While a systematic approach and human centered development process can go a long way toward eliminating error, the compl ete exclusion of operator error is not a reasonable expectation. The ISS environment in particular poses chal lenging conditions, especially for flight controllers and astronauts. Operating a scientific laboratory 250 miles above the Earth is a complicated and dangerous task with high stakes and a steep learning curve. While human error is a reality that may never be fully eliminated, smart implementation of carefully chosen tools and techniques can go a long way toward minimizing risk and increasing the efficie ncy of NASA's space science operations.
\end{abstract}

\section{Introduction}

$\mathrm{T}$ hroughout history, humans have proven themselves to be both capable of incredible achievement and susceptible to catastrophic errors. Between 2005 and 2007, the National Motor Vehicle Crash Causation Survey analyzed a sample of 5,470 automobile crashes in the United States and determined that $94 \%$ of the total crashes could be attributed to human error. ${ }^{1}$ In 2014, IBM's yearly Cyber Security Intelligence Index revealed that $95 \%$ of cyber security incidents investigated for the report involved human error. ${ }^{2}$ And in a study of NASA mishaps between 1996 and $2005,57 \%$ of type $\mathrm{A}^{\ddagger}$ mishaps were attributed to human error. ${ }^{3}$ Despite their tendency to commit errors, humans remain an integral component of many systems, including payload operations aboard the International Space Station (ISS). Fortunately, typical limitations on human perception, cognition, and physical performance have been well studied and documented. However, $24 \times 7$ flight operations present additional, unique challenges to human operators. Factors common to space operations environments such as stress and fatigue have proven to further limit human information processing and decision making capabilities in individual and unpredictable ways. Because of these and other limitations on performance capabilities, human errors are inevitable in all systems with human components. The relevant question becomes how to best address human error as a component of ISS payload operations, where errors are often costly to critical science objectives.

\footnotetext{
** Payload Communications Manager, Training and Crew Operations Branch, NASA Marshall Space Flight Center/EO20, Huntsville, Alabama, USA. samantha.s.harris@nasa.gov

$\dagger$ Payload Operations Director, Operations Directors Office, NASA Marshall Space Flight Center/EO03, Huntsville, Alabama, USA. beau.c.simpson@nasa.gov

$¥$ A Type A Mishap is a mishap resulting in one or more of the following: (1) an occupational injury or illness resulting in a fatality, a permanent total disability, or the hospitalization for inpatient care of 3 or more people within 30 workdays of the mishap; (2) a total direct cost of mission failure and property damage of \$1 million or more; (3) a crewed aircraft hull loss; (4) an occurrence of an unexpected aircraft departure from controlled flight (except high performance jet/test aircraft such as F-15, F-16, F/A-18, T-38, OV-10, and T-34, when engaged in flight test activities).
} 


\section{Humans Make Mistakes}

At its core, human performance is limited by the capability of the physical human body. Human eyes can only see to a certain distance. Human ears can only hear within certain frequencies. Human brains can only process so much information simultaneously. Beyond physical limitations, human performance is further complicated by the more personal aspects of the human experience. Temporary feelings and conditions like emotion, stress, and fatigue significantly impact the performance of human operators in unique ways. Due to the constrained and variable nature of human performance, no amount of effort will eliminate human error entirely in systems with human elements. However, studying and developing an understanding of the various limits on human capability and the factors that influence human behavior can enable us to better predict and eliminate many human errors in space operations.

\section{A. Visual and Auditory}

To make decisions and solve problems, humans must first take in information from their surroundings. For the average operator, vision serves as the primary source for this information. Human vision can roughly be broken down into two stages; physical reception of stimuli, and interpretation and processing of stimuli. When it comes to physical reception, the structure of the human eye dictates the way humans perceive characteristics like size, depth, brightness, and color. Understanding how the structure of the eye impacts human abilities to perceive visual information can help us to eliminate some human errors in operations. For example, we know that cones, one of the two primary types of photoreceptors in the retina, are more densely packed toward the center of the visual field. This concentration of cones makes it easy for humans to focus on a stimulus at the center of our vision, but difficult to read or distinguish stimuli at increasing distances from that focal point. Rods, the other type of photoreceptors in the retina, are concentrated in the outer part of the visual field and are sensitive to change. This concentration of change-sensitive rods enables the average human to see one specific type of stimulus well in peripheral vision: movement ${ }^{4}$. Therefore, as designers and operators, we can use this knowledge to decrease the odds that a human operator will miss a stimulus in their peripheral vision by introducing motion into that stimulus.

In order to keep up with the vast number of stimuli physically perceived by our eyes each day, humans rely on a variety of visual processing techniques. One technique that humans use to resolve ambiguity is reliance on expectations. While our expectations generally enable us to correctly interpret the information that we see, relying on expectations sometimes causes us to make errors. For example, is the text in Figure 1 correct? While human perception systems tend to be efficient, they are also prone to error.

To supplement information gathered via the visual system, humans use the auditory system. The human auditory system allows the body to collect sounds and interpret them into meaningful messages. The human ear is limited by what frequencies it is capable of detecting, and if sounds are too loud or frequencies are too

\section{The lazy orange cat sleeps under the the cozy armchair.}

\author{
Figure 1. Proofreader's Illusion.
} similar, humans are unable to differentiate sound. In a world of remote operations, operators at the POIC are more reliant on their sense of hearing than average workers. Face to face operations with astronauts and scientists are often not possible, so a significant amount of information is exchanged solely over voice channels (referred to as loops). Most operators monitor many loops simultaneously. For example, the Payload Communications Manager (PAYCOM) monitors an average of approximately fifteen loops including four primary Space to Ground (S/G) loops and many ground loops. Studies have shown that humans are capable of fully processing only one stream of auditory information at a time. When operators are asked to monitor multiple loops simultaneously, the auditory system filters out background information (or noise) in order to focus on important information (or signals). When there are multiple simultaneous conversations on the loops, operators must cope with what is referred to as a low signal-to-noise ratio. Low signal-to-noise ratios have been shown to result in increased frequency of human error. ${ }^{5}$

\section{B. Movement}

After a stimulus is received through sensory receptors such as the vision or auditory system, the information is transmitted to the brain. The brain processes the information and sends commands to the appropriate muscles to respond to the stimulus. This total time required for a human to respond to a stimulus can be broken down into reaction time and movement time. Both reaction time and movement time depend on a variety of factors and are subject to limitations in human capability. For example, there is a lower limit on how quickly a human operator is capable of recognizing that a command needs to be sent, and physically clicking the button to send the command. By effectively 
designing operator tools and environments with human factors in mind, it is possible to minimize movement time. However, a human operator will never be capable of instantaneous action. It is important that we consider these and additional limitations (such as reach envelopes, repetitive movements, fatigue, etc.) on human operator capability as we design tools, tasks, and processes in our operations environment. For example, an operator will be able to send a command more quickly if the tools he must use to do so are comfortably within his reach envelope. If the operator must wheel his chair down his desk a few feet and reach for a mouse to send the command, the time required to complete the task will be longer. A great deal of work has been done in the field of ergonomics that can be used to assist with increasing efficiency and minimizing error by ensuring tools, tasks, and procedures are appropriately designed for operators.

\section{Memory}

The human memory system is used to store and retrieve information. It is generally accepted that there are three types of human memory: sensory memory, short-term or working memory, and long-term memory. Sensory memory is the shortest term element of memory and is used to store information received through the senses. Information stored in sensory memory is either re-written as more information arrives or moved from sensory memory into short term memory through attention. Attention is the process used by humans to filter out what is important from the vast amount of information in sensory memory at any given time. Humans are able to focus attention selectively, but are limited in the number of stimuli they can attend to simultaneously.

Short-term or working memory is used for temporary recall of information. Short-term memory can be accessed rapidly, but it also decays quickly and is limited in capacity. It is relatively well known that the average person is limited to recalling $7 \pm 2$ chunks of information using short term memory. This seems relatively simple, but a lot of research has been done on short term memory, and it can be quirky. Things like the recency effect, or the tendency for humans to better recall items seen more recently than those in the middle of a list, and interference have been shown to impact short term memory under certain circumstances ${ }^{4}$.

Long-term memory is used for the long-term storage of information and is where we store everything we know. Long-term memory takes longer to access than short-term memory, but has a huge, if not unlimited, capacity. Information is moved from short to long-term memory through rehearsal, and many factors such as the total time spent learning, the distribution of practice sessions through time, and the meaningfulness of the information impact the efficiency of the transition ${ }^{4}$. After transition to long-term memory, information is subject to decay and interference. Information is retrieved from long-term memory through recognition or recall, with recall being the more difficult of the two.

Operators at the POIC rely heavily on memory to recall factual information as well as knowledge of actions and procedures. For example, the Operations Controller (OC) is required to recall a wide variety of documentation including Payload Regulations and Flight Rules that govern payload operations. While not expected to recall everything they have read, OCs are expected to recognize situations that fall under the jurisdiction of existing documentation. This is a difficult job that requires efficient and effective recognition and recall abilities. Human memory is limited under the best of circumstances, and the flight control environment provides especially challenging conditions. Many conditions commonly experienced by flight controllers, such as stress, anxiety, and sleep deprivation, have been shown to negatively impact memory. Sleepdeprivation specifically has been repeatedly proven to negatively impact both working memory and attention. ${ }^{6}$

\section{Reasoning and Problem Solving}

After information is received and stored, humans utilize that information to reason and solve problems. These thinking processes can be complex and introduce many opportunities for error. One notoriously unreliable technique humans commonly use is called inductive reasoning. Inductive reasoning involves generalizing from cases seen to infer information about cases unseen. Since the number of cases seen is usually limited, inductive reasoning is a useful process that humans use out of necessity to draw conclusions about the rest of the world. However, inductive reasoning has a high error rate ${ }^{4}$. For example, a flight controller who has witnessed only false fire alarms may infer that the next fire alarm he hears is false as well. The next time there is a true fire event, he may respond less diligently to the alarm due to his incorrect inductive reasoning. 
Another tool humans use to decode the world around them is mental models. Mental models are the theories individuals develop to explain the behavior of situations or systems. Mental models are especially dangerous because they tend to be partial, unstable, and unscientific. For example, an astronaut may develop a mental model that payloads are powered on by placing the power switch in the up position because most payloads he has seen operate this way. If he is asked to operate a payload that is inconsistent with this model, the astronaut may be more likely to commit an error and place the switch in the incorrect position. A real example of this can be seen with the Minus Eighty-Degree Lab Freezer for ISS (MELFI) racks onboard the ISS. For MELFI racks located in the US Lab, the rack is powered on by flipping the Rack Power Switch (RPS) down as shown in Figure 2. For MELFI racks located in the Japanese Experiment Module (JEM), the rack is powered on by flipping the RPS up.

One final opportunity for error that will be discussed in this abbreviated list is the way humans acquire skills. As humans reach mastery of a particular skill, that behavior has a tendency to become automatic. Automatic behavior can be efficient, but results in errors when the context of the activity changes without the operator noticing. For example, a PAYCOM who is accustomed to using S/G 2 to call astronauts aboard the space station may fail to notice that $S / G$ 2 has been privatized for a medical conference and make an erroneous call on that channel out of habit.

\section{E. Affect}

The above sections have discussed human perceptual and cognitive abilities under the implied assumption that humans operate at full capability most of the time. In truth, human performance and error are much more complicated than that. Humans experience a wide range of feelings that are unique to individuals at specific moments in time. Affect comprises emotions (such as anger and frustration), moods (such as cheerfulness), and dispositional traits (such as competitiveness or optimism). A lot of research has been done on the impact affect has on performance, and that research has revealed that affect impacts many skills necessary for successful operations including decision making, creativity, prosocial behavior, negotiation, conflict resolution, and leadership effectiveness. ${ }^{7}$ These findings suggest that even the most proficient flight controllers are susceptible to the effects of emotions or moods manifesting within their work quality. For example, an operator who consistently earns excellent performance ratings may be pulled over by a traffic officer and receive a speeding ticket on the way into work resulting in a negative attitude that hinders her decision making for the duration of her shift.

\section{F. Stress}

Another factor that has been shown to negatively impact human perceptual and cognitive performance is stress. Studies have linked increased stress levels to decreased performance in tasks that utilize memory, especially when utilization of working memory or retrieval of information stored in memory is required. Stress has also been linked to decreased performance in tasks that require divided attention or decision making. ${ }^{8}$ Stress tends to be an especially difficult condition to quantify and control because it is a highly individualized experience. Conditions that result in stress induced errors for one operator may not impact the performance of another. For flight controllers, stress levels vary widely and change by the minute. For example, a Stowage engineer may work many hours with no significant events, only to be caught completely off guard by a call from an astronaut who cannot find a tool in the provided location. The Stowage officer then immediately finds himself under immense pressure to find a workaround for the problem by providing alternative locations or backup options for the missing item as quickly as possible to preserve available crew time.

\section{G. Fatigue}

There is a great deal of literature describing the link between fatigue and cognitive impairment. In general, research has consistently associated insufficient sleep with "cognitive problems, mood alterations, reduced job performance, reduced motivation, increased safety risks, and physiological changes." Many of the most notable disasters in history, including the Three Mile Island nuclear disaster, the Exxon Valdez oil spill, and the loss of NASA's space shuttle Challenger, have been attributed, at least in part, to human error due to sleep deprivation and fatigue..$^{10}$ In a $24 \times 7$ 
space operations environment where operators work schedules that require rotational sleep shifting, fatigue is an unavoidable reality. In addition to fatigued ground operators, fatigue of the astronaut crew operators onboard the space station must be considered. A 2014 study of 64 astronauts from 80 space shuttle missions and 21 astronauts from 13 ISS missions concluded that "sleep deficiency was prevalent not only during space shuttle and ISS missions, but also throughout a 3 month preflight training interval." 11

\section{H. Additional Error Producing Factors}

Additional research in human error has focused on the specific features of the tasks humans complete. In 1986, J.C. Williams developed the Human Error Assessment and Reduction Technique (HEART) to calculate the probability that a human error will occur during the performance of a particular task. The HEART, which is still used today, accounts for the impact of a list of pre-defined Error Producing Conditions (EPCs) on task performance. HEART EPCs represent conditions that have been proven through research to result in human performance errors and include many conditions common in space operations. Some of the most significant EPCs relevant to space operations include: unfamiliarity with a low frequency event, time

Table 1. Summary of Error Producing Conditions ranked in order of known effect. ${ }^{12}$

\begin{tabular}{lc}
\hline Condition & Risk factor \\
\hline Unfamiliarity with the task & $(\times 17)$ \\
Time shortage & $(\times 11)$ \\
Poor signal:noise ratio & $(\times 10)$ \\
Poor human system interface & $(\times 8)$ \\
Designer user mismatch & $(\times 8)$ \\
Irreversibility of errors & $(\times 8)$ \\
Information overload & $(\times 6)$ \\
Negative transfer between tasks & $(\times 5)$ \\
Misperception of risk & $(\times 4)$ \\
Poor feedback from system & $(\times 4)$ \\
Inexperience - not lack of training & $(\times 3)$ \\
Poor instructions or procedures & $(\times 3)$ \\
Inadequate checking & $(\times 3)$ \\
Educational mismatch of person with task & $(\times 2)$ \\
Disturbed sleep patterns & $(\times 1 \cdot 6)$ \\
Hostile environment & $(\times 1 \cdot 2)$ \\
Monotony and boredom & $(\times 1 \cdot 1)$ \\
\hline
\end{tabular}
shortage for error detection and correction, low signal-to-noise ratio, operator inexperience, a mismatch between the mental models of the operator and the designer, a lack of obvious means to reverse unintended actions, information overload, a need to transfer specific knowledge from task to task without loss, ambiguity in performance standards, and impoverished quality of information conveyed by person to person interaction. ${ }^{5}$ Table 1 provides a summary of some EPCs ranked in order of known effect per HEART guidelines. These examples represent only a small portion of all EPCs that may impact a typical shift for a payload operator. However, even this small list sufficiently illustrates the difficulty of reducing human error in a space operations environment.

\section{Preventing Errors Before They Occur}

The first and most effective line of defense that should be implemented against human error is prevention. While it will never be possible to prevent all human errors from occurring, it is possible to improve upon current statistics by changing the way we think about human error. Dr. James Reason, a leading psychologist in the field of human error, has identified two primary paradigms that may be used when addressing the problem of human error: the person approach and the system approach. ${ }^{13}$ In the person approach, blame is placed on the individual who committed the error and a moral shortcoming of the operator such as forgetfulness, inattention, or lack of motivation is identified as the root cause of the error. Efforts to prevent similar errors from recurring are targeted at attempting to reduce variation in individual operator behavior through doing such things as requiring additional training, developing new procedures, publicly shaming those who do make mistakes, and sometimes even threatening termination for mishaps. While this approach has historically been preferred in the United States, it has some significant drawbacks that must be considered. First, a person approach to error discourages error reporting and creates a culture of blame and secrecy. Second, this approach fails to identify a root cause that can be reliably fixed. Human performance, by nature, is constrained and variable, and no amount of threats or training will prevent humans from making errors. While aperson approach to error is sometimes necessary to correct recurring abnormal or harmful behavior patterns in specific individuals, attempts to achieve complete control over human behavior will always prove futile.

The alternative to the person approach, which the POIC has strived to increasingly adopt as we work to combat human error, is the system approach. In the system approach, human operators are assumed to be fallible and expected to err. The systems approach shifts focus away from the behavioral shortcomings of individual operators and instead addresses the external, upstream conditions that allow errors to occur. Errors are viewed as systematically connected to the features of operators' tools and tasks rather than their behavior alone. Operator behavior is difficult to change, but tools and tasks are much simpler to reliably correct. In the systems approach, root cause analyses are conducted 
to determine the true cause of individual errors, and the root causes are addressed in ways that prevent similar errors from being made again by any operator in the future. While it is not possible to engineer out variability in operator behavior, it is possible to increase the robustness of systems so that variability no longer results in errors. The systems approach is one of constant vigilance and continuous improvement that minimizes errors in organizations by systematically eliminating opportunities for those errors to occur.

\section{Data Gathering}

To effectively prevent errors in organizations, we must first understand where those errors are occurring in our unique system. To understand what kind of errors are occurring within our operations, the POIC uses tools including the POIC Error Log shown in Figure 3 and the Payload Action Item List (PAIL). When a flight controller commits an error, the individual is asked to fill out an Error Report through the Error Log. Error reports contain inform ation about the console discipline of the operator who committed the error, the date and time of the error, and a written description of the error. The Error Report is then sent to the team lead of the discipline who committed the error, and the team lead becomes responsible for conducting the root cause analysis and supplying a resolution for the report. PAILs are used to document anomalies in payload products that must be corrected before that product is used again. For example, a PAIL

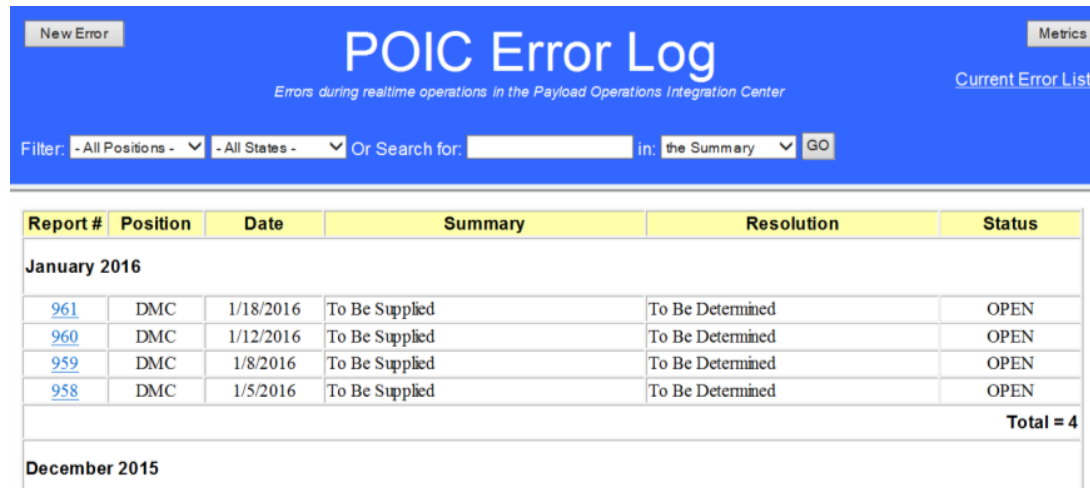

Figure 3. POIC Error Log. would be submitted if the crew onboard the Space Station reported an error in a written procedure.

There is no one size fits all approach to gathering error data, and individual approaches must be tailored to specific organizations and industries. However, one of the most critical steps in gathering accurate data in any organization is the creation of a safe and just reporting culture. Employees must have faith that "the boss can hear bad news" and reporting their own errors benefits the greater good of the organization. If errors result in consequences viewed by employees as unjust, most errors that can be covered up will not be reported. Without an accurate account of mishaps and close calls, risk management becomes more difficult for the organization because patterns indicating systematic issues may not be discovered until problems become widespread or catastrophic. The POIC error reporting system has enabled the identification and correction of many common operator and payload errors. However, culture change is still required for these efforts to reach full effectiveness. A stigma on error reporting remains, and many operators are hesitant or unwilling to submit reports unless requested to by management. Due to this stigma, errors are not always reported honestly and many opportunities for systematic organizational improvement are missed.

In addition to error reporting systems, debriefs are a useful tool for gathering data on errors and close calls. In many industries, for example military aviation, debriefs are standard practice. Within the Space Station program, it is common practice to debrief "Lessons Learned" after each flight increment and after significant but uncommon or offnominal events. It is also standard practice within the POIC to debrief all simulated training events. These debriefs have played a significant role in the identification of error patterns and creation of opportunities for systematic improvement in payload operations. However, debriefs have proven difficult to integrate into the current real time operations model at the POIC. Managers at the POIC have often discussed the potential benefits of team debriefs after each real time console shift ends, but logistical difficulties have so far been determined to outweigh anticipated benefits.

\section{J. Analysis and Prevention Measures}

Once the organization has a better understanding of where errors and close calls are occurring, root cause analyses should be conducted to determine why those errors are occurring. Often, human error patterns tend to follow the wellknown Pareto principle (also known as the 80-20 rule), with states that approximately $80 \%$ of effects come from $20 \%$ of causes. Said another way, a study of specific errors in an organization is likely to reveal that around $80 \%$ of human errors are caused by $20 \%$ of causes. Many errors will likely be traced back, at least in part, to operator limitations and error producing conditions described in section II of this paper. Payload operations onboard the ISS are multifaceted and may be broken down into many different types of operations and errors. 


\section{Command Errors}

One source of error in payload operations is command errors. Command errors occur when an operator sends an incorrect command to the ISS or fails to send a command at the appropriate time. To better understand the root causes of command errors in ISS spaceflight operations, NASA conducted a Human Error Analysis that included data from the ISS Command Error Database (CED) at Johnson Space Center (JSC). While this database does not track errors in payload commands from the POIC, it does provide data for commands being sent to the ISS from a $24 \times 7$ operations environment similar to the POIC and is therefore a useful analogy. The NASA study analyzed data on 414 errors out of 746,790 total commands captured between August 2004 and June 2008. Investigators categorized the self-reported contributing factors for those errors as shown in Figure 4. The most often cited contributing factors were cognitive overload and available time, which were cited in $22 \%$ and $21 \%$ of errors, respectively. Additional contributing factors included complacency (13\%), stress/fatigue $(10 \%)$, procedures $(7 \%)$, team collaboration $(2 \%)$ and training/experience $(2 \%) .{ }^{14}$

Since commanding tasks are software based, operator errors can often be effectively addressed through targeted human factors improvements to existing software tools. For example, a recently

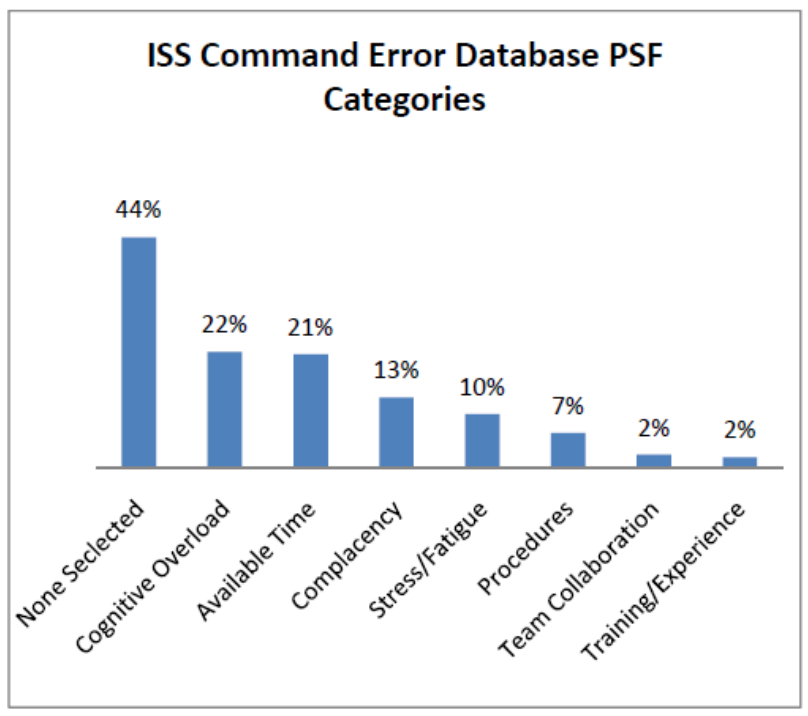

Figure 4. Error Data Broken Down by Category from the ISS Command Error Database. ${ }^{14}$ reported error involved an incorrect command being sent to the ISS. An analysis determined that the command sent in error was obsolete, so that command was removed from the operator display entirely. Removing obsolete commands from displays may not prevent all command errors, but it does ensure those specific commands will not be sent in error by any operator again. In another incident, an operator failed to re-start health and status polling to a payload after an off-nominal event. The root cause analysis revealed that power data for payloads in that location was not included in the operator's console display. The missing information was added to the display during the next update, and the error has not occurred again.

For commands deemed to be critical, the POIC employs redundant verification to prevent operator error. Before sending a critical command, the commander must obtain permission from the Payload Operations Director (POD) who obtains permission from the ISS Flight Director. After permission is received, the commander must enable the command, select the command, and verify the command before it is sent to the vehicle. After a critical command is sent, it is automatically disabled to protect against redundant delivery. For both critical and non-critical commanding operations, verification techniques including software prompts like the one in Figure 5 have proven to be a valuable defense against errors. They provide operators the option to undo unintended actions without causing damage to systems. Multiple teams within the POIC have eliminated operator errors through the use of redundant operator verification within the chain of command and targeted implementation of human factors tools such as verification prompts in software

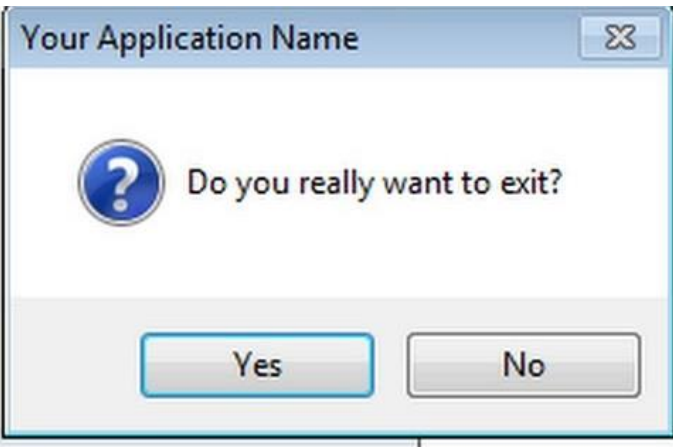

Figure 5. Verification Prompt. applications.

Another technique the POIC has employed to minimize human error is automation. The Payload Rack Officer (PRO) team is one of the most commanding-heavy teams at the POIC and takes an especially proactive systems approach to commanding errors and automation. The PRO team identifies patterns of recurring errors using the Error Log, and develops scripts that guide operators through commanding sequences that are most likely to result in operator errors. These scripts automate commands when possible and use prompts to guide operators through coordination and 
decision making tasks that cannot be automated. Command scripts have proven especially useful for sequences of commands that are always performed in the same order. For example, PROs utilize a script to power on specific chambers within the lab freezers. Command scripts have successfully been used to minimize or eliminate many of the most common payload command errors at the POIC. However, automation introduces its own unique challenges. Automation may cause operators to become complacent and less situationally aware, which reduces their ability to respond to unexpected events in real time. Also, it is important that operators maintain the knowledge nece ssary to recover when automated tools fail to perform as expected.

\section{Communication Errors}

Another source of operator error within payload operations, ineffective communication, tends to be more difficult to quantify and systematically prevent. While command errors can often be addressed using software solutions, communication based tasks are more heavily interpersonal and less amenable to technical solutions. The operations model at the POIC is designed around multiple flight control discipline teams who each specialize in a payload system and work together to operate payloads owned by remotely located Payload Developers (PDs) and Principal Investigators (PIs). Successful payload operations require extensive and efficient communication both within the real time flight control room and remotely with payload owners and back room specialists. A $24 \times 7$ operations model also requires shift handovers to communicate relevant information between off-going and on-coming console operators. Systematic standardization of such tasks has proven difficult due to the impact personality and communication styles have on the way individual operators perform them. Recent examples of payload operations errorsultimately attributed to communication errors include: an error in a crew stowage product due to miscommunication with a PD, failure to communicate the need for uplink of a real time product from a back room to the flight control team, and failure to hand over information between shifts.

Payload operations necessitate the timely transfer of large amounts of diverse information between many stakeholders. To ensure that the information gets to the proper places at the proper time, it is important to establish standardized processes for information routing and control. For example, when a crew member onboard the space station leaves a written note for the payload operations team, the PAYCOM is responsible for disseminating that information to the proper stakeholders. A formal Standard Operating Procedure (SOP) provides instructions for how this information should be shared. SOPs are established for many processes and establish consistency, responsibility, and accountability for communication practices. When processes are not standardized (or standardized processes are not followed), performance standards may be ambiguous to operators, thus resulting in greater error frequency. Errors may also occur when communications processes are used inconsistently, or when information is communicated using multiple uncontrolled processes. For example, information passed from back room support specialists to real time flight controllers is a recurring source of error at the POIC. While a root cause has not been identified, these errors are likely at least partly caused by inconsistency in how this information is communicated. For example, at one point in time, real time PAYCOMs were required to check at least three separate information sources (including email, handover notes, and a daily planning report) to determine whether a back room support specialist had identified any topics for the morning Daily Planning Conference.

When possible, the POIC utilizes software tools to help standardize information routing, for example during shift handover. Shift handovers require significant knowledge transfer between operators and, while necessary, introduce opportunities for communication errors. To help mitigate communication errors from shift handovers, each cadre position is expected to keep a log of pertinent information throughout their shift. Specific log entries that on-coming operators need to be aware of or possibly work during their shift, are color coded using position specific flagging systems as shown in Figure 6. Flagging a $\log$ entry as a "handover item" alerts on-coming operators that they will need to take action pertaining to that specific log entry during their shift. Log entries flagged as handover items also serve as reminders for the offgoing operator to verbally discuss the open itemin person with the on-coming operator before checking out for the

day. In addition to communicating information across handovers, console logs are a useful tool for communicating real time operations information to other stakeholders.

\begin{tabular}{|c|c|c|c|}
\hline Log / Entry: & POD Test Log / 1851291 & \multicolumn{2}{|c|}{$\begin{array}{l}\text { Subject: H\&S } \quad \text { Payload: Enter Payload name (Payload A) } \\
\text { PRO reported that Payload A is no longer sending Health and Status (H\&S) to the ground } \\
\text { he believes the data connector may have been bumped or disconnected somehow. } \\
\text { Since we are in crew sleep we will ask the crew to have a look in the morning. }\end{array}$} \\
\hline GMT. & 2016/074 21:09:11 & \multirow{3}{*}{\multicolumn{2}{|c|}{$\begin{array}{l}\text { PRO reported that Payload A is no longer sending Health and Status (H\&S) to the ground, } \\
\text { he believes the data connector may have been bumped or disconnected somehow. } \\
\text { Since we are in crew sleep we will ask the crew to have a look in the morning. } \\
\text { Orbit } 2 \text { suggest asking the crew to check data cable at ER1O2 locker } 5 \text { location. }\end{array}$}} \\
\hline Flag: & Handover & & \\
\hline Author: & Simpson, Beau C. & & \\
\hline Log / Entry: & POD Test Log / 1851290 & Subject: Handover Complete & Payload: \\
\hline GMT: & 2016/074 21:06:49 & \multirow{3}{*}{\multicolumn{2}{|c|}{ Simpso }} \\
\hline Flag: & & & \\
\hline Author: & Simpson, Beau C. & & \\
\hline & & \multicolumn{2}{|c|}{$\begin{array}{l}\text { This entry and the entries above are only examples of how we log at the } \\
\text { POIC for Space Ops paper } 2016\end{array}$} \\
\hline
\end{tabular}

Figure 6 Logging Tool with Handover Flag. 
Another software tool used to assist with communication is the Stancil recorder. Most real time operations communication is done over voice loops. Flight controllers are required to monitor many loops at a time, which can result in a low signal-to-noise ratio and challenge the capabilities of the human auditory and information processing systems. The Stancil recorder automatically records voice traffic on most loops, and provides a backup solution by allowing operators to playback information that is not fully understood during initial calls. Operators can search for clips by time or loop, and can save and send specific clips to other operators or back room support specialists as necessary.

Checklists may be either software based or simply written on a piece of paper, and may be used to encourage consistent, repeatable, and error-free operations. Checklists are standard practice in many high hazard, high consequence industries like aviation, medical care, and manufacturing. They are popular because of their demonstrated ability to minimize risk and error, and improve efficiency and performance outcomes. ${ }^{15}$ Many of the most efficient payload operators use checklists, but currently, a widespread checklist culture does not exist within the POIC. While consistent checklist implementation will not eliminate all errors, it would help mitigate the effects of many of the error producing conditions payload operators must cope with daily.

Real time payload operations are constantly changing and often unpredictable. Standardized procedures and checklists are important tools, but cannot account for the wide variety of potential outcomes. To cope such uncertainty,

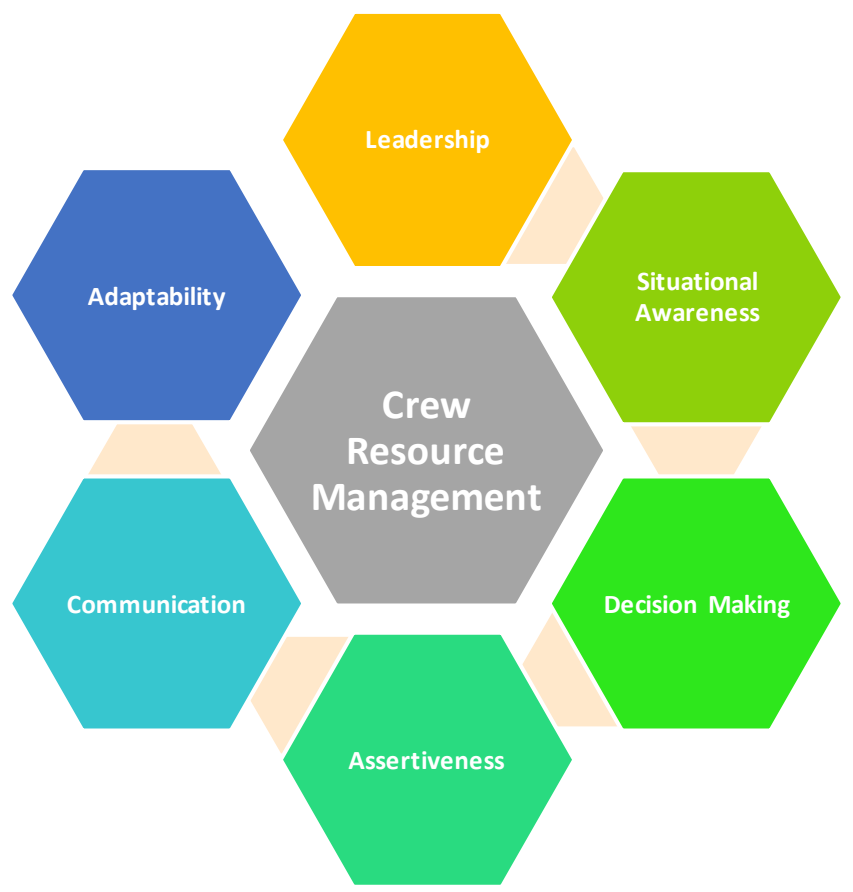

Figure 7 Foundations of Crew Resource Management. human operators themselves must be trained to serve as a defense against error. To effectively prepare operators for such challenging jobs, a technique called Crew Resource Management (CRM) may be useful. Pioneered by NASA research in response to a series of aviation disasters in the 1970s, CRM focuses on improving operator performance through emphasis on the interpersonal and cognitive skills necessary to manage resources in complex systems. Skills targeted for improvement through CRM include communication, leadership, situational awareness, teamwork, conflict resolution, stress management, and decision making. CRM encourages cooperation through leadership at all levels, and seeks to empower all operators to speak up when necessary. In highly technical industries like space payload operations, the "soft skills" emphasized by CRM are sometimes neglected in favor of emphasis on technical skills. This is a loss, because while technical skills are important, operators thoroughly trained in both technical and CRM skills are more empowered in systems requiring interpersonal cooperation, and are likely to be better prepared to achieve positive outcomes despite error producing conditions in the workplace.

\section{Hardware Errors}

For operations that require the use of tools or hardware, the hardware itself may be considered an important tool in error prevention. Clever implementation of human factors theories and techniques in the hardware design phase can go a long way toward elimination of operator error during the operations phase. One example of such a technique is poka- yoke mistake proofing. The term poka-yoke was coined in Japan by an engineer working at Toyota in the 1960s and is still in wide use in industrial engineering today. Essentially, poka-yoke devices prevent, correct, or clearly draw attention to operator errors as they occur. Poka-yoke devices are especially useful in hardware that will be operated by astronaut crews. One example of poka-yoke hardware currently used onboard the space stationis keyed connectors. Keyed connectors may only be assembled one way, the correct way, thereby preventing operator errors by disallowing incorrect assembly.

Because payloads are designed and manufactured by a wide variety of payload developers, standardizing payload hardware to human factors design criteria can be a challenge. To assist payload developers as they design hardware, the Space Station program provides a variety of resources. The Human Factors Implementation Team (HFIT) includes 
astronaut representatives and human factors experts, and helps identify potential human factors issues early in the hardware design process. The Astronaut Office also provides consultation and feedback for hardware operability differences that may be encountered in microgravity. For example, payload developers may not consider that fasteners and screws are difficult to keep track of in microgravity. An expert in microgravity operations may suggest the use of captive fasteners, like the one shown in Figure 8, to prevent fastener loss and preserve crew time. The ISS Payload Label Approval Team (IPLAT) ensures consistency in crew interface payload labeling to facilitate crew understanding of hardware. For payloads that include crew interfaces with graphic user displays, the Payload Displays Review Team (PDRT) performs usability testing and suggests ways interfaces should be improved. Taking the proper time to design hardware for usability now is an investment that can greatly reduce the risk of operator errors that may result in science loss later.

Human factors and usability should also be considered in the design of hardware tools that will be utilized by operators on the ground. Tools should be designed to support operators in the tasks they are performing, and should be developed using an iterative process that incorporates user feedback into the design. Common usability techniques like color coding, auditory cues, standardization with familiar systems, elimination of unnecessary options, and undo capability have all proven useful in payload operations. When errors occur, the results of root cause analyses should be fed back to hardware design teams who have the ability to improve interfaces through updates or redesigns. Sometimes, operators themselves configure their personal tool setups to prevent errors. For example, one of the most significant PAYCOM errors is accidental transmission over space to ground. To prevent accidental selection of a space to ground loop, many PAYCOMs have configured their loop selection panel to separate the space to ground loops from other commonly used loops. This minimizes the chance that a PAYCOM will accidentally select a space to ground loop in error.

While the POIC has successfully taken strides toward implementing a system approach to human error, there is still room to improve. Root cause analyses for error reports are typically performed by individual discipline teams with few standard processes or requirements. Consequently, the approach taken by individual analysts varies widely between the person and systems approaches. Many errors attributed to human error have been resolved with statements such as: operator will pay more attention next time, operator was reminded of the proper protocol, will continue training and stress accuracy of products, or no corrective action necessary. Unfortunately, resolutions like these fail to address the root causes of the errors and do little to prevent future operators from repeating them. In a dynamic operations environment, there is always risk that an error that is harmless under one set of conditions may result in significant impact upon recurrence. To achieve continued positive improvement in human error rates at the POIC, a systems approach must consistently be applied to all root cause analyses so underlying causes of errors are effectively identified and addressed.

\section{Responding to Errors}

A consistent, systematic approach to error prevention is a powerful tool that can be used to minimize or eliminate many common operator errors. However, some level of operator error will always remain and must be accepted as an operational constraint. Assuming that some errors will slip through even the best prevention systems, organizations should consider how to best prepare for and respond to those errors that do occur. To effectively minimize the negative impacts of these errors, systems must have the ability to do three things: identify that an error has occurred, reduce the impact of the error, and recover from the error.

\section{K. Identify Errors}

To effectively respond to an error, operators must first recognize that an error has occurred. For tasks with end items that can be monitored by software, detection of off-nominal conditions caused by errors is relatively rapid at the POIC. Operators utilize a variety of software tools to constantly monitor health and status parameters for racks and payloads that generate visual cues and alert tones when parameters go out of limits. Many of the software based tools used for identification of off-nominal conditions, for example POIC's Exception Monitoring tool, utilize a combination of human factors friendly elements like auditory cues and color coding to help operators identify and 
rapidly process error messages. Another tool is Fault Summary, which is used to alert console operators and station crew to off-nominal events onboard the space station. Fault Summary codes notifications for emergencies red, warnings pink, cautions yellow, and advisories blue. Color coding systems like these allow operators to more rapidly assess the criticality of error messages that would otherwise take longer to process. When minimized, Fault Summary automatically pops up from the task bar any time a new message populates in the display. This motion effectively grabs the attention of operators, even from their peripheral vision.

Errors that cannot be immediately identified by software may be more difficult to detect. Some errors are obvious, for example an accidental transmission on space to ground. Other errors, like an incorrect location code provided for an item in a stowage note, may not be noticed until an astronaut attempts to execute the activity and reports them. For errors like these, checklists can be useful tools to help identify steps that have been missed.

\section{Reduce Impact}

After errors are made, focus should switch to minimizing the immediate impact of the error on the system. Efforts to reduce the impact of operator errors should be both proactive, targeting errors that have not yet occurred, and reactive, targeting errors that have already occurred. One technique commonly used to proactively minimize the impact of future errors is fault tolerance. Fault tolerance allows systems to continue to operate nominally after experiencing one or more failures, and should be designed into hardware and processes whenever feasible. Fault tolerant systems should be robust enough to accommodate the variability of human behavior, and able to endure one or more operator errors without impact to end products or customers. At the POIC, fault tolerance is generally achieved through redundancy. For example, in an effort to preserve valuable astronaut crew time, all products uplinked for use by the crew go through a formal, multi-layer review and approval process. Products are reviewed by enough reviewers that errors missed by one reviewer are usually caught by another. The redundancy in this process allows for individual reviewers to make isolated errors without compromising the quality of the end product. The Space Station program also utilizes redundant hardware whenever possible, and many systems onboard have some combination of hot and cold backups. Fault tolerance does not prevent operator errors, but it does prevent those errors from negatively impacting the operability of the system.

For errors that occur despite our best proactive efforts, a reactive approach to mitigate their impact becomes necessary. In a dynamic system like payload operations, the first step in containing impacts is identifying them. In some cases, impacts caused by errors may be obvious, but in cases where the impacts are varied or complex, identifying and quantifying those impacts may be difficult and time consuming. In real time operations environments, conditions are constantly changing and impacts of errors may be additionally complicated by time or path dependency. These impacts may vary widely in severity depending on the specific conditions present at the time of impact, and an error that causes no impact at one time may result in a significant impact at another due to condition changes. For example, a power cycle to a facility that provides services to multiple payloads has the potential for either very low or very high impact depending on the state of the individual payloads in that rack. At one moment, all the payloads in the rack may be dormant and indifferent to a power cycle. At another moment, a power cycle may result in complete loss of science for one or more of those payloads. In off-nominal events, operators at the POIC are trained to determine the impacts to their systems and report them to the POD as soon as possible.

After the POD compiles the list of impacts, he must decide how to most efficiently and effectively minimize damage caused by those impacts. In payload operations, this process typically includes real time stakeholder analysis coupled with consultation of documentation of pre-determined priorities. Priority is generally given to successful completion of science objectives, but tradeoffs must often be made with regard to stakeholder considerations such as astronaut crew time, station resources such as power and cooling, impacts to international partners, and impacts to other science experiments. In situations like these, established operations documents like Flight Rules and Payload Regulations are valuable tools. Documentation provides a consistent, pre-determined impact reduction method for many situations. Another important technique used to reduce the impact of errors in operations is workarounds. Workarounds, or temporary fixes used to accomplish goals, allow the operations team to continue to execute payload operations despite noticeable failures or impacts. For example, a lost tool may be substituted with a different but similar tool as a workaround to preserve science and keep operations on schedule for the day.

\section{Recover}

Once short-term impacts are contained and workarounds are implemented, the final step in error response is recovering from the error. Ideally, recovery is accomplished quickly by the real time operations team. However, in some cases, recovery efforts may be handed over to the next shift or to a back room support team if recovery is not time critical, requires additional expertise or extensive cross-organizational coordination, or detracts from other critical real time operations. Recovery efforts may have a combination of technical and non-technical elements, and 
often require follow up with stakeholders. For errors reported to the ground by the crew, it is sometimes necessary to follow up with a resolution. Scope and methods of recovery effort vary widely based on the nature of the error and severity of impact, but they should always include documentation. Error documentation is critical for historical recording, and an important source of lessons learned for the future. Documentation also informs a thorough root cause analysis of the error, which enables the organization to explore ways to prevent the error from happening again by instituting a systems approach to error prevention.

\section{Conclusion}

On an orbiting laboratory where critical science opportunities may not be repeatable and scarce resources are often not replaceable, the margin for error is unforgiving. Every operator error poses a risk to science outcomes and resources, and should be addressed with due diligence. There is no one size fits all method for addressing operator error, but the POIC has discovered that some practices are especially useful in space payload operations. A consistent, systems approach to human error prevention that emphasizes continuous improvement of operator tools and processes is vital to eliminate opportunities for error. Human factors and usability techniques including automation, information routing and control, standardization, and iterative design processes that include user feedback have been successfully employed to reduce operator errors in payload operations. Operators proficient in interpersonal and leadership skills such as those emphasized by Crew Resource Management provide an additional line of defense against error, and are better equipped to minimize the impact of errors that do occur. Operating a laboratory 250 miles above Earth is a unique challenge that provides endless opportunities to battle human error in the pursuit of excellence in payload operations. 


\section{References}

${ }^{1}$ Singh, S., "Critical reasons for crashes investigated in the National Motor Vehicle Crash Causation Survey," DOT HS 812 $115,2015$.

2"IBM Security Services 2014: Cyber Security Intelligence Index,” IBM Corporation, 2014.

${ }^{3}$ Chandler, F., Chang, Y.H., Mosleh, A., Marble, J., Boring, R., and Gertman, D., "Human Reliability Analysis Methods: Selection Guidance for NASA," NASA, 2006.

${ }^{4}$ Dix, A., Finlay, J., Abowd, G.D., and Beale, R., Human-Computer Interaction, $3^{\text {rd }}$ ed., Pearson Education Limited, Essex, England, 2004, Chap. 1.

${ }^{5}$ Williams, J.C., "A data-based method for assessing and reducing human error to improve operational performance," Proceedings of the IEEE Fourth Conference on Human Factors and Power Plants, Vol. 1, IEEE, New York, 1988, pp.436-450.

${ }^{6}$ Alhola, P., and Polo-Kantola, P., "Sleep deprivation: Impact on cognitive performance," Neuropsychiatric Disease and Treatment, Vol. 3, No. 5, Oct. 2007, pp. 553-567.

${ }^{7}$ Barsade, S .G., and Gibson, D.E., "Why Does Affect Matter in Organizations?," Academy of Management Perspectives, Feb. 2007, pp. 36-59.

${ }^{8}$ LeBlanc, V .R., "The Effects of Acute Stress on Performance: Implications for Health Professions Education," Academic Medicine, Vol. 84, No. 10, Oct. 2009, pp. S25-S33.

${ }^{9}$ Rogers, A.E., Patient Safety and Quality: An Evidence-Based Handbook for Nurses., $1^{\text {st }}$ ed., Agency for Healthcare Research and Quality (US), Rockville, MD, 2008, Chap. 40.

${ }^{10}$ Mitler, M. M. et al, “Catastrophes, Sleep, and Public Policy: Consensus Report,” Sleep, Vol. 11, No. , Feb 1988, pp. 100109.

${ }^{11}$ Barger, L.K. et al., "Prevalence of sleep deficiency and use of hypnotic drugs in astronauts before, during, and after spaceflight: an observational study," The Lancet Neurology, Vol. 13, No. 9, Aug, 2014, pp. 904-912.

${ }^{12}$ Reason J., “Understanding adverse events: human factors,” Quality in Health Care, Vol. 4, No. 2, Jun 1995, pp. 80-89.

${ }^{13}$ Reason, J., "Human error: models and management,” Western Journal of Medicine, Vol. 172, No. 6, Jun 2000, pp. $393-396$.

${ }^{14}$ Chandler, F., Heard, I.A., Presley, M., Burg, A., Mitten, E., and Mongan, P., "NASA Human Error Analysis,” NASA, 2010.

${ }^{15}$ Hales B. M. and Pronovost, P. J., "The checklist--a tool for error management and performance improvement," J Crit Care, Vol. 21, No. 3, Sep 2006, pp. 231-235. 